\title{
Role of nitric oxide in hypoxia-induced hyperventilation and hypothermia: participation of the locus coeruleus
}

\section{G. Fabris ${ }^{1}$, \\ J.A. Anselmo-Franci ${ }^{2}$ and L.G.S. Branco 2}

\author{
${ }^{1}$ Departamento de Fisiologia, Faculdade de Medicina de Ribeirão Preto and \\ 2Departamento de Morfologia, Estomatologia e Fisiologia, \\ Faculdade de 0 dontologia de Ribeirão Preto, Universidade de São Paulo, \\ Ribeirão Preto, SP, Brasil
}

\section{Correspondence \\ L.G.S. Branco \\ Departamento de Fisiologia \\ Faculdade de 0 dontologia de \\ Ribeirão Preto, USP \\ 14040-904 Ribeirão Preto, SP \\ Brasil \\ Fax: + 55-16-633-0999 \\ E-mail: branco@forp.usp.br \\ Presented at the Meeting "NO Brazil, Basic and Clinical Aspects of Nitric O xide", \\ Foz do Iguaçu, PR, Brazil, \\ March 10-13, 1999. \\ Research supported by FAPESP and CNPq. G. Fabris was the recipient of a postgraduate fellowship from FAPESP.}

Received June 17, 1999

Accepted September 9, 1999

\section{Abstract}

Hypoxia elicits hyperventilation and hypothermia, but the mechanisms involved are not well understood. The nitric oxide (NO) pathway is involved in hypoxia-induced hypothermia and hyperventilation, and works as a neuromodulator in the central nervous system, including the locus coeruleus (LC), which is a noradrenergic nucleus in the pons. The LC plays a role in a number of stress-induced responses, but its participation in the control of breathing and thermoregulation is unclear. Thus, in the present study, we tested the hypothesis that LC plays a role in the hypoxia-induced hypothermia and hyperventilation, and that NO is involved in these responses. Electrolytic lesions were performed bilaterally within the LC in awake unrestrained adult male Wistar rats weighing 250-350 g. Body temperature and pulmonary ventilation $\left(\mathrm{V}_{\mathrm{E}}\right)$ were measured. The rats were divided into 3 groups: control $(\mathrm{N}=16)$, sham operated $(\mathrm{N}=7)$ and LC lesioned $(\mathrm{N}=19)$, and each group received a saline or an $N^{\mathrm{G}}$-nitro-L-arginine methyl ester (LNAME, $250 \mu \mathrm{g} / \mu \mathrm{l}$ ) intracerebroventricular (icv) injection. No significant difference was observed between control and sham-operated rats. Hypoxia ( $7 \%$ inspired $\mathrm{O}_{2}$ ) caused hyperventilation and hypothermia in both control (from $541.62 \pm 35.02$ to $1816.18 \pm 170.7$ and $36.3 \pm 0.12$ to $34.4 \pm 0.09$, respectively) and LC-lesioned rats (LCLR) (from $694.65 \pm 63.17$ to $2670.29 \pm 471.33$ and $36 \pm 0.12$ to $35.3 \pm 0.12$, respectively), but the increase in $\mathrm{V}_{\mathrm{E}}$ was higher $(\mathrm{P}<0.05)$ and hypothermia was reduced $(\mathrm{P}<0.05)$ in LCLR. L-NAME caused no significant change in $\mathrm{V}_{\mathrm{E}}$ or in body temperature under normoxia, but abolished both the hypoxia-induced hyperventilation and hypothermia. Hypoxia-induced hyperventilation was reduced in LCLR treated with L-NAME. L-NAME also abolished the hypoxia-induced hypothermia in LCLR. The present data indicate that hypoxia-induced hyperventilation and hypothermia may be related to the $\mathrm{LC}$, and that $\mathrm{NO}$ is involved in these responses.
Key words

- Nitric oxide

- Locus coeruleus

- Hypoxia

- Ventilation

- Body temperature

- Rat 


\section{Introduction}

Hypoxic activation of arterial chemoreceptors increases the excitatory synaptic drive of respiratory neurons in vivo (1). An increase in central respiratory activity also occurs in in vitro preparations in which chemoreceptor afferents are deleted $(2,3)$. Hence, activation of medullary chemosensory neurons (4) or direct stimulation of respiratory neurons (2) contributes to the increased respiratory response to hypoxia.

Moreover, oxygen consumption and body temperature decrease during acute exposure to hypoxia in a variety of animal species (5). Although the precise nature for this hypoxic hypothermia is not fully understood, it is generally admitted that it results from a reduction in the thermoregulatory set point, which has been referred to as anapyrexia (6), rather than from a reduction in body temperature due to an impairment of the thermoregulatory effector mechanisms caused by a limitation in oxygen availability (7). The importance of this response is emphasized by reports that show an increase in survival of the tested species if they are allowed to become hypothermic during hypoxia exposure (8).

Sympathetic activation is one of the major components of the adaptive responses to oxygen reduction, especially in the regulation of cardiovascular events that accompany hyperventilation (9-11). Sympathetic neurons receive both excitatory and inhibitory influences from noradrenergic cell groups located in the brainstem (12). The locus coeruleus (LC), a pair of nuclei located in the pons, is an assembly of densely packed noradrenergic neurons; its extensive projections provide noradrenergic innervation to many brain areas and to the spinal cord (13). Hypoxia causes a significant increase in $c$ fos in the LC (14). Although little is known about the central pathways and neuromodulators involved in the neuro-physiological adjustments occurring during hypoxia, some candidates have been proposed, including nitric oxide (15).

The diffusible lipophilic gas nitric oxide (NO) has been recognized as a physiological molecule because of its role in body temperature regulation $(16,17)$. A previous study indicated that the hypoxia-induced hypothermia depends on the NO pathway (17). Also, NO may serve as a neurotransmitter in the central nervous system (CNS) and inhibit inhibitory synaptic transmission that is triggered by CNS hypoxia (15).

The purpose of the present study was to test the hypothesis that the LC participates in the control of body temperature and pulmonary ventilation $\left(\mathrm{V}_{\mathrm{E}}\right)$ under normoxic and hypoxic conditions, and that $\mathrm{NO}$ is involved in this process in the CNS.

\section{Material and Methods}

\section{Animals}

Experiments were performed on adult male Wistar rats weighing 250-350 g, housed at controlled temperature $\left(25 \pm 2^{\circ} \mathrm{C}\right)$ and exposed to a daily 12:12-h light-dark cycle. The animals were allowed free access to water and food. Experiments were performed between 10:00 a.m. and 3:00 p.m.

Animals were divided into three groups: control $(\mathrm{N}=16)$, sham-operated $(\mathrm{N}=7)$ and LC-lesioned rats (LCLR) $(\mathrm{N}=19)$. The experimental group comprised rats submitted to bilateral lesion within the LC. Each group was divided into 2 other subgroups: 1) animals which received an intracerebroventricular (icv) injection of saline $(1 \mu \mathrm{l})$ and 2$)$ animals which received an $i c v$ injection of $N^{\mathrm{G}}$-nitro-L-arginine methyl ester (L-NAME, $250 \mu \mathrm{g} / \mu \mathrm{l}$; Sigma Chemical Co., St. Louis, MO, USA).

\section{Surgery}

Rats used for LC lesion were submitted to general anesthesia with 2,2,2-tribromoethanol (Aldrich, Milwaukee, WI, USA) and 
mounted in a Kopf stereotaxic apparatus. Bilateral electrolytic lesions (2 mA, $10 \mathrm{~s}$, positive electrode) were made using a constant current source and coated stainless steel electrodes in the LC (coordinates: A -3.4 $\mathrm{mm}$ from lambda, $\mathrm{L} \pm 1.2 \mathrm{~mm}, \mathrm{D} 6.8 \mathrm{~mm}$ ) (18). Sham-operated rats were similarly prepared, but the electrode was placed $2 \mathrm{~mm}$ above the dorsal coordinate and no current was passed through the electrodes. This distinction was made to test the possibility of alteration in body temperature and $\mathrm{V}_{\mathrm{E}}$ induced by the surgical intervention. Experiments were initiated $24 \mathrm{~h}$ or seven days after lesion.

At the end of the experiment, the animal was killed with ether and the brain was removed and post-fixed in formol saline $(10 \%)$ for 1 week. After fixation, the brainstem was embedded in paraffin. Serial 13$\mu \mathrm{m}$ frozen sections were cut and stained by the Nissl method for histological verification of the location and extension of the lesions (or their absence in the case of shamoperated rats) and schematically presented in neuroanatomic maps (19). The LC lesions comprised a necrotic core surrounded by a zone of gliosis tissue. The sham-operated rats had an identifiable electrode tract, but no discernible lesion. Only the animals with 50 to $100 \%$ bilateral LC lesion were used for analysis of the results.

Rats used for $i c v$ injection of L-NAME were also anesthetized with 2,2,2-tribromoethanol and fixed in a Kopf stereotaxic apparatus. A stainless steel guide cannula (14 $\mathrm{mm}$ ) was implanted into the right lateral ventricle of the brain (coordinates: A -1.0 $\mathrm{mm}$ from bregma, $\mathrm{L} \pm 1.6 \mathrm{~mm}, \mathrm{D}-3.2$ to -3.7 $\mathrm{mm})(18)$. The displacement of the meniscus in a water manometer ensured correct positioning of the cannula in the right lateral ventricle. The cannula was attached to the bone with stainless steel screws and acrylic cement. A tight-fitting stylet was kept inside the guide cannula to prevent occlusion. The surgical procedures were performed over a period of $40 \mathrm{~min}$. Six days after cannula placement, the animals were submitted to LC lesion or sham operation.

\section{Experimental protocol}

Experiment 1. Determination of the combined effect of lesion and hypoxia on body temperature and $V_{E}$. Experiments were performed using animals first exposed to humidified room air and then to a humidified hypoxic mixture of 7\% $\mathrm{O}_{2}$ (AGA, Sertãozinho, SP, Brazil). $\mathrm{V}_{\mathrm{E}}$ was determined by the plethysmograph method (20), and body temperature was determined by inserting a thermocouple probe into the colon. Before the experiment, the animals were habituated to temperature measurements in order to avoid stress-induced elevations in body temperature.

Each animal was placed in a 5-liter plexiglass chamber and allowed to move about freely while the chamber was flushed with humidified air. After the animals remained calm (at least $60 \mathrm{~min}$ ), control body temperature was measured. Control $\mathrm{V}_{\mathrm{E}}$ was measured and the test gas mixture $(7 \%$ inspired $\mathrm{O}_{2}$ ) was flushed through the chamber for $60 \mathrm{~min}$. Ventilation was also measured after 5, 15, 45 and 60 min of exposure to hypoxia, and body temperature was measured at the end of the hypoxic period. The chamber was then flushed with humidified air for 30 min and during this period $V_{E}$ was measured at 15-min intervals. At the end of the experiment, body temperature was measured again.

During the ventilation measurements, the flow was interrupted and the chamber sealed for short periods of time, and the oscillations in air temperature caused by breathing were measured as pressure oscillations. Calibration for volume was obtained during each measurement by injecting the chamber with a known amount of air $(0.2 \mathrm{ml})$ using a graduated syringe. Signals from a differential air transducer displayed on a paper re- 
corder (HP) allowed the calculation of respiratory frequency $\left(\mathrm{f}_{\mathrm{R}}\right)$ and tidal volume $\left(\mathrm{V}_{\mathrm{T}}\right)$ by appropriate correction factors (21).

Experiment 2. Determination of the combined effects of NOS blocker and hypoxia on body temperature and $V_{E}$. Rats were treated with an icv injection of L-NAME or saline. A 705-LT, 50- $\mu$ l Hamilton syringe and a Mizzy dental injection needle (200 $\mu \mathrm{m}$ OD) were used for $i c v$ injections. Sterile saline was the vehicle in which L-NAME was dissolved, in a final volume of $1 \mu$ l. The injection was performed over a period of $2 \mathrm{~min}$, and $1 \mathrm{~min}$ was allowed to elapse before the injection needle was removed from the guide cannula, to avoid reflux. The same protocol described above was then used.

Experiment 3. Determination of the combined effects of LC lesion, NOS blocker and hypoxia on body temperature and $V_{E}$. Six days after cannula placement, animals were submitted to LC lesion or sham operation and the same protocol as described above, with an icv injection of L-NAME or saline, was used.

\section{Statistical analysis}

Data are reported as means \pm SEM. Changes in body temperature, $V_{T}, f_{R}$ and $V_{E}$ were evaluated by ordinary analysis of variance (ANOVA) or ANOVA for repeated measures to analyze the temporal effect of hypoxia on body temperature and $\mathrm{V}_{\mathrm{E}}$. The differences between means were assessed by the Tukey-Kramer multiple comparisons test. Values of $\mathrm{P}<0.05$ were considered to be significant.

\section{Results}

In all experimental protocols, the mean chamber temperature was $25.41 \pm 0.52^{\circ} \mathrm{C}$ (SEM), and the room temperature was 24.55 $\pm 0.47^{\circ} \mathrm{C}$. Figure 1 shows the lesion sites of individual rats $(\mathrm{N}=7)$.

During normoxia and the recovery period after hypoxia, body temperature and $\mathrm{V}_{\mathrm{E}}$ did not differ between groups. Also, body temperature and $\mathrm{V}_{\mathrm{E}}$ during the return to air
Figure 1 - Representative drawings showing the localization of the locus coeruleus $(\mathrm{LC})(\mathrm{A})$ and lesions (dark areas, B) placed bilaterally in the nucleus. Sequential sections of seven different animals.
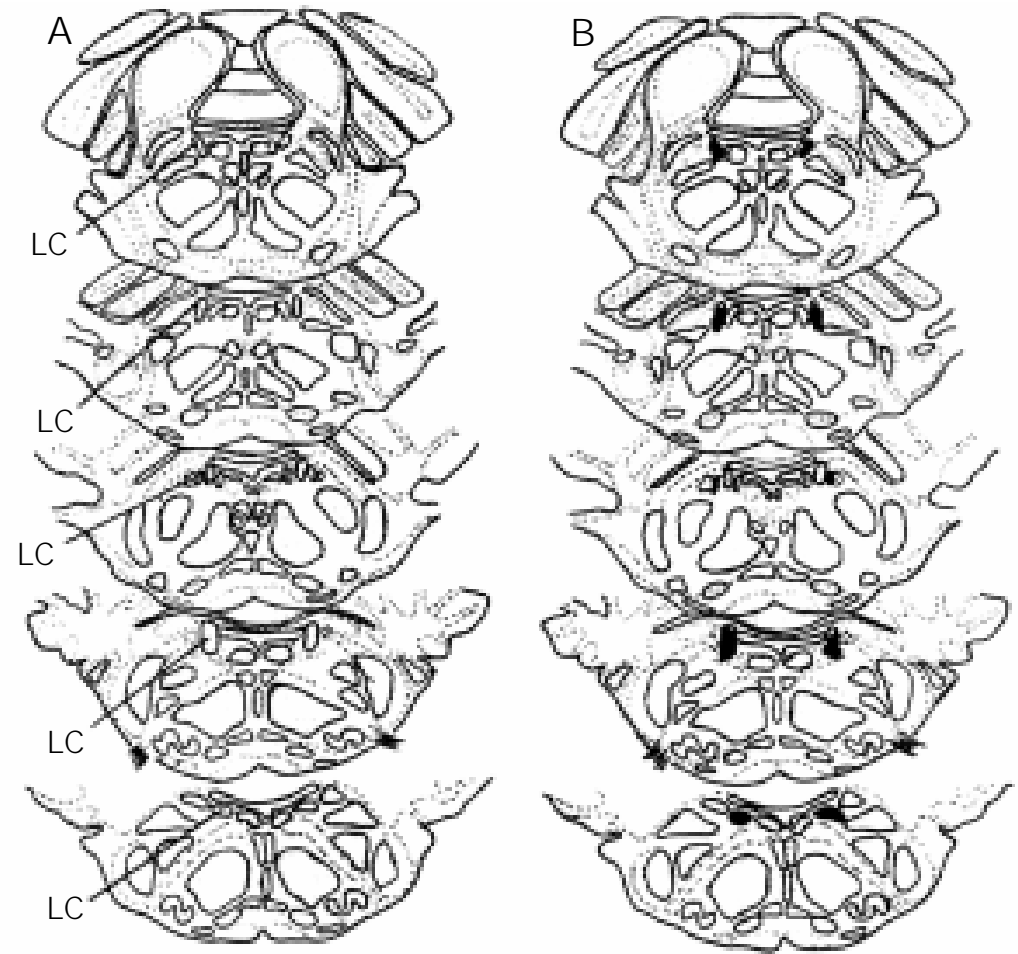

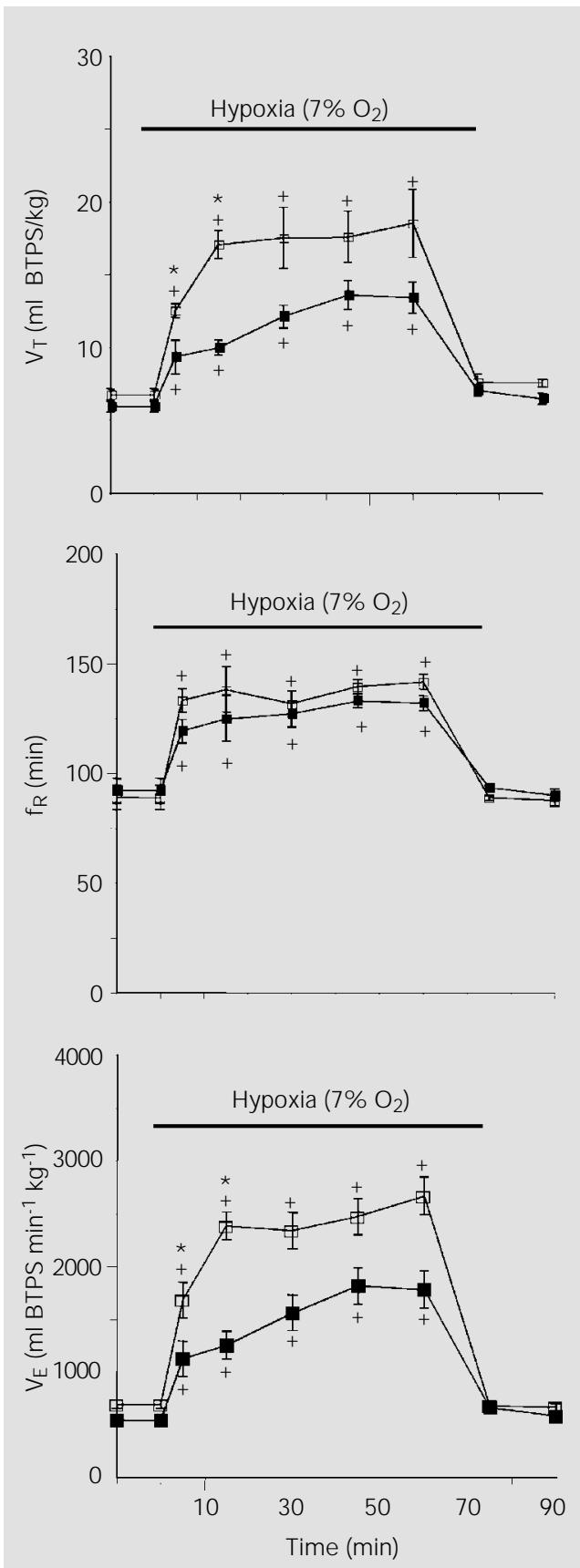

Figure 2 - Effects of hypoxia on tidal volume $\left(V_{T}\right)$, respiratory frequency $\left(f_{R}\right)$ and pulmonary ventilation $\left(V_{E}\right)$ of control rats (filled squares) $(\mathrm{N}=16)$ and LC-lesioned rats (open squares) $(N=7)$. Values are reported as means \pm SEM. The hypoxia-induced hyperventilation was higher in LC-lesioned rats. +Significant increase $(P<0.05)$ in $V_{T}, f_{R}$ and $V_{E}$ after hypoxia compared to normoxia. *Significant increase in $\mathrm{V}_{\mathrm{T}}$ and $\mathrm{V}_{\mathrm{E}}$ of LClesioned rats compared to control group. BTPS, Body temperature, pressure, saturated with water vapor.

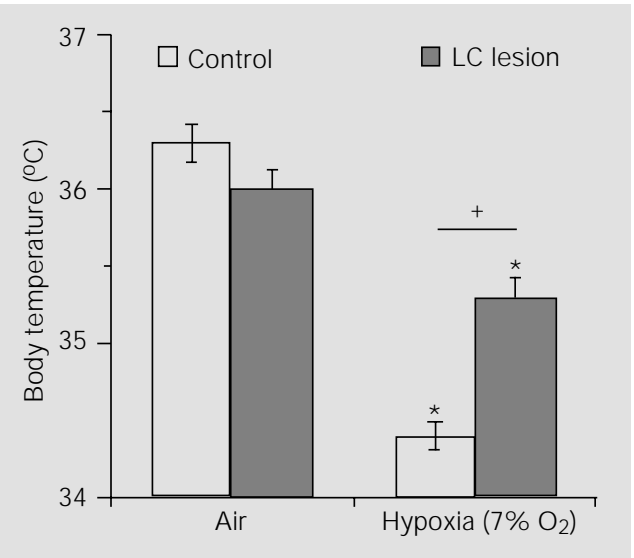

Figure 3 - Effects of hypoxia on body temperature of the control group $(\mathrm{N}=16)$ and locus coeruleus (LC)-lesioned rats $(\mathrm{N}=7)$. Values are reported as means \pm SEM. * Significant reduction $(\mathrm{P}<0.05)$ of mean body temperature after hypoxia. +Significant reduction $(\mathrm{P}<0.05)$ in the magnitude of hypoxia-induced hypothermia. after hypoxia did not differ from the baseline value in any group. The sham-operated group did not differ significantly from control animals in any variable (data not shown). Data obtained from LCLR 1 week after lesion did not differ from control or sham-operated rats (data not shown).

Experiment 1. Combined effect of lesion and hypoxia on body temperature and $V_{E}$. Figure 2 shows the effect of hypoxia on $\mathrm{V}_{\mathrm{T}}$, $f_{R}$ and $V_{E}$, in control and LCLR. When inspired $\mathrm{O}_{2}$ was reduced from 21 to $7 \%$, a significant $(\mathrm{P}<0.05)$ increase in ventilation was observed in both groups. The increase in $\mathrm{V}_{\mathrm{E}}$ was significantly higher $(\mathrm{P}<0.05)$ in LCLR than in control rats at 5 and $15 \mathrm{~min}$ of exposure to hypoxia. The hypoxia-induced hyperventilation of LCLR was primarily the result of a significant elevation in $\mathrm{V}_{\mathrm{T}}$, rather than a change in $f_{R}$, which did not differ from control. The breathing pattern was recovered during return to air, when the experimental and control groups showed similar recordings.

Figure 3 shows the effect of hypoxia on body temperature of control and LCLR. After hypoxia, a significant decrease in body temperature was observed in both control $(\mathrm{P}<0.05)$ and LCLR $(\mathrm{P}<0.05)$, but the hypoxia-induced hypothermia was reduced in LCLR. After hypoxia, the drop in body temperature was significantly lower $(\mathrm{P}<0.05)$ in LCLR compared to control.

Experiment 2. Combined effects of NOS 
Figure 4 - Combined effect of hypoxia and intracerebroventricular injection of $\mathrm{N}^{\mathrm{G}}$-nitro-L-arginine methyl ester (L-NAME; $250 \mu \mathrm{g} / \mu \mathrm{l})$ on tidal volume $\left(\mathrm{V}_{\mathrm{T}}\right)$, respiratory frequency $\left(f_{R}\right)$ and pulmonary ventilation $\left(V_{E}\right)$ in $L-$ NAME group (open squares) (N $=7$ ) and saline group (filled squares) $(\mathrm{N}=7)$. Values are reported as means \pm SEM. ${ }^{+}$Significant increase $(P<0.05)$ in $V_{T}$, $f_{R}$ and $V_{E}$ of the saline group after hypoxia compared to normoxia. *Significant difference $(P<0.05)$, after hypoxia, between L-NAME-treated rats and salinetreated rats. BTPS, Body temperature, pressure, saturated with water vapor. blocker and hypoxia on body temperature and $V_{E}$. Figure 4 shows that the combination of saline injection and hypoxia caused a significant increase in $\mathrm{V}_{\mathrm{E}}$, similar to that obtained by application of hypoxia only. When L-NAME was injected $30 \mathrm{~min}$ before hypoxia, hypoxia-induced hyperventilation was abolished, a fact primarily due to a
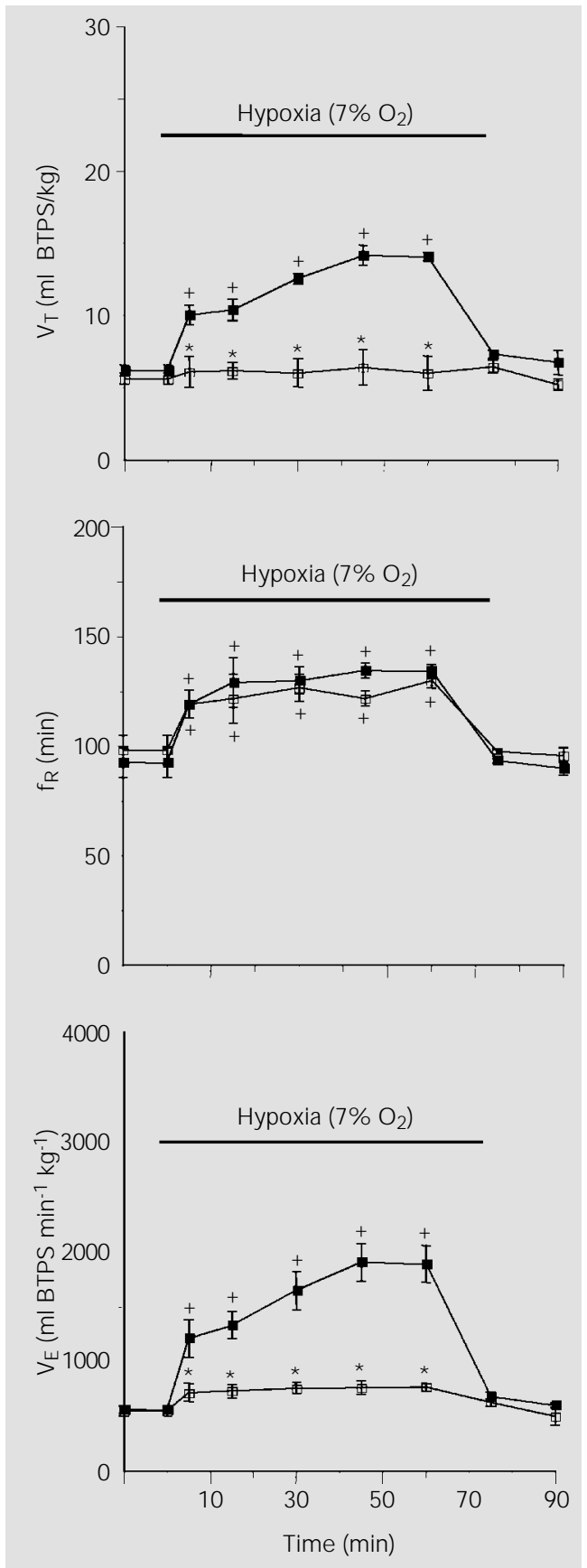

significant reduction in $\mathrm{V}_{\mathrm{T}}$ after hypoxia, rather than a change in $f_{R}$.

Hypoxia failed to induce a reduction of body temperature when L-NAME was given intracerebroventricularly. These data are plotted in Figure 5.

Experiment 3. Combined effects of $L C$ lesion, NOS blocker and hypoxia on body temperature and $V_{E}$. Figure 6 shows a significant increase $(\mathrm{P}<0.05)$ in $\mathrm{V}_{\mathrm{E}}$ in LCLR + saline and LCLR + L-NAME after hypoxia, compared to normoxia, which was significantly higher $(\mathrm{P}<0.05)$ in $\mathrm{LCLR}+$ saline than in LCLR + L-NAME, at 5 and 15 min of exposure to hypoxia. The reduced hypoxiainduced hyperventilation of the LCLR + LNAME group was primarily due to a significant reduction in $\mathrm{V}_{\mathrm{T}}$ after hypoxia, rather than a change in $f_{R}$.

Figure 7 shows that the combination of LC lesion, saline and hypoxia caused a significant $(\mathrm{P}<0.05)$ drop in body temperature, similar to that obtained by LC lesion and hypoxia only. When L-NAME was injected

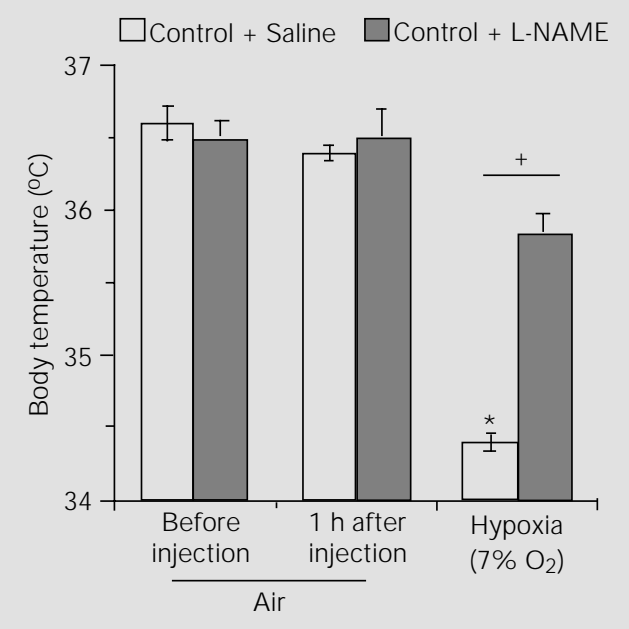

Figure 5 - Combined effect of hypoxia and intracerebroventricular (icv) injection of $\mathrm{N}^{\mathrm{G}}$-nitro-L-arginine methyl ester (L-NAME; $250 \mu \mathrm{g} / \mu \mathrm{l}$ ) on body temperature of saline- and L-NAM E-treated rats ( $\mathrm{N}=7$ for both groups). Values are reported as means \pm SEM. *Significant drop $(\mathrm{P}<0.05)$ in body temperature after hypoxia of saline group compared to normoxia. +Significant difference $(P<0.05)$ in the magnitude of hypoxia-induced hypothermia. 

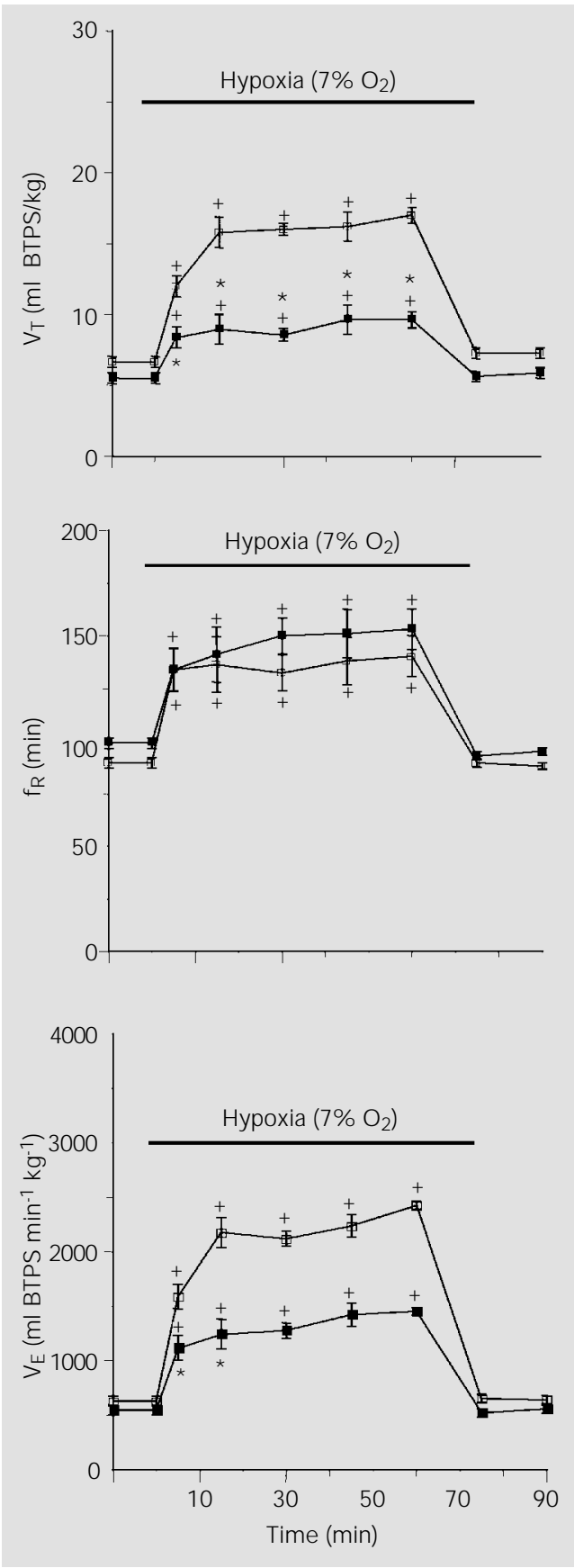

Figure 6 - Combined effect of hypoxia and intracerebroventricular (icv) injection of $\mathrm{N}^{\mathrm{G}}$-nitro-L-arginine methyl ester (L-NAME; $250 \mu \mathrm{g} / \mu \mathrm{l})$ on tidal volume $\left(\mathrm{V}_{\mathrm{T}}\right)$, respiratory frequency $\left(f_{R}\right)$ and pulmonary ventilation $\left(V_{E}\right)$ of LClesioned rats that received an icv injection of saline (open squares) $(\mathrm{N}=5)$ or L-NAME (filled squares) $(\mathrm{N}=7)$. Values are reported as means \pm SEM. +Significant increase $(P<0.05)$ in $V_{T}, f_{R}$ and $V_{E}$ after hypoxia compared to normoxia. *Significant difference $(P<0.05)$ in $\mathrm{V}_{T}$ and $V_{E}$ of L-NAME-treated rats compared to saline. BTPS, Body temperature, pressure, saturated with water vapor.

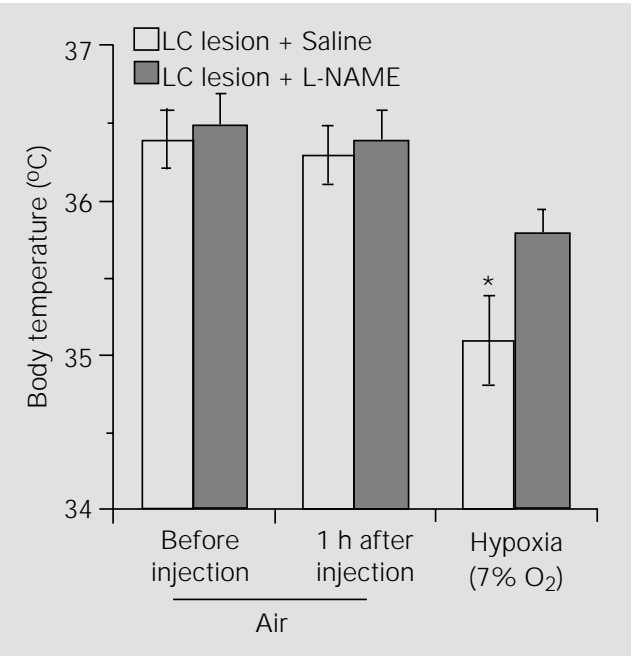

Figure 7 - Combined effect of hypoxia and intracerebroventricular injection of $\mathrm{N}^{\mathrm{G}}$-nitro-Larginine methyl ester (L-NAME; $250 \mu \mathrm{g} / \mu \mathrm{l})$ on body temperature of locus coeruleus (LC)-lesioned rats that received an icv injection of saline $(\mathrm{N}=5)$ or L-NAME $(\mathrm{N}=7)$. Values are reported as means \pm SEM. * Significant drop $(\mathrm{P}<0.05)$ in body temperature after hypoxia.

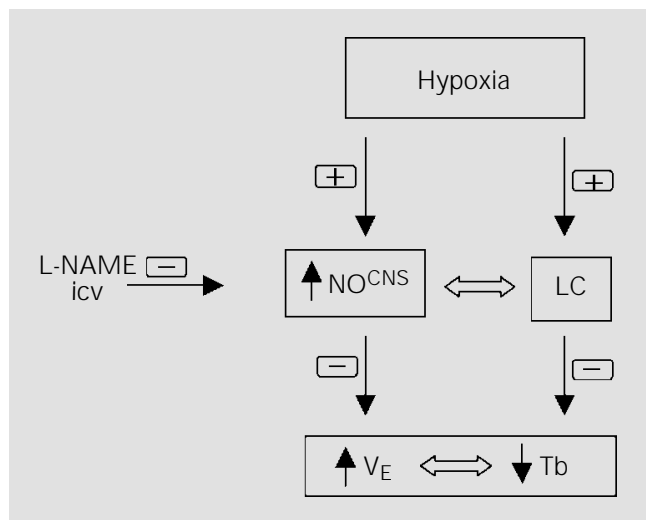

Figure 8 - Possible mechanisms through which the locus coeruleus (LC) and nitric oxide (NO) could mediate hypoxia-induced hyperventilation and hypothermia. Hypoxia leads to an increase in NO in the CNS and in the firing rate of LC neurons, which cause an increase in pulmonary ventilation $\left(\mathrm{V}_{\mathrm{E}}\right)$ and a reduction in body temperature (Tb). $N^{G}$-nitro-L-arginine methyl ester (L-NAME) prevents hypoxia-induced hyperventilation and hypothermia by inhibiting NO synthesis.

30 min before hypoxia in LCLR, hypoxiainduced hypothermia was abolished, as observed by application of L-NAME in control rats. However, there was no significant difference in body temperature between LCLR + saline and LCLR + L-NAME after hypoxia.

\section{Discussion}

This study provides evidence that the LC participates in the control of pulmonary ventilation and body temperature during hypoxia challenge, since bilateral electrolytic lesions of the nucleus caused an increased ventilatory response to hypoxia and a reduced hypoxia-induced hypothermia. A possible link between the two responses cannot 
be excluded since a drop in body temperature leads to a reduced ventilatory drive. These data suggest that LC plays an inhibitory role in the hypoxia-induced hyperventilation and an excitatory role in the hypoxia-induced anapyrexia. Moreover, NO seems to play an important role in these responses to oxygen deprivation, since $i c v$ injection of L-NAME abolished both hypoxia-induced hyperventilation and hypothermia. Additionally, the inhibitory role of LC in pulmonary ventilation may depend on the NO pathway, since LCLR treated with L-NAME had a reduced ventilatory response to hypoxia.

In a wide variety of animal species, hypoxia elicits a number of compensatory responses, including increased ventilation and cardiac output (5). Decreases in $\mathrm{PaO}_{2}$ are monitored by peripheral arterial chemoreceptors that evoke excitation of chemosensory fibers projecting in the brainstem within the nucleus tractus solitarius (NTS) (12). In the brainstem, these afferent inputs are processed and integrated together with other inputs to yield a final command to the respiratory motoneurons resulting in an increase in respiratory drive (22). However, the increased ventilation is $\mathrm{O}_{2}$ consuming, a fact that limits its beneficial effect. Hypoxia also elicits a decrease in body temperature and oxygen consumption (7), which is considered to be an adaptive response because it decreases $\mathrm{O}_{2}$ demand according to the $\mathrm{Q}_{10}$ effect, promotes a leftward shift of the oxyhemoglobin dissociation curve, and blunts the energetically costly response to hypoxia, e.g., increased cardiac output and ventilation (5). Previous studies have demonstrated that hypoxia per se causes hypothermia in a variety of organisms ranging from protozoans to mammals (23), but only recently did the mechanisms responsible for the hypoxia-induced anapyrexia start to be suggested.

The role of CNS in body temperature control is subjected to numerous modifiers, such as lactate, adenosine and histamine (for review, see 23), but none of the possible candidates can trigger a complete hypothermic response. Nitric oxide works as a physiological messenger molecule that may serve as a neurotransmitter in the CNS (24). NO in the CNS may have an important role in the hypoxia-induced hypothermia since during inhibition of the NO pathway hypoxia failed to reduce body temperature (17). Besides mediating hypoxia-induced hypothermia, oxygen deprivation was recently shown to lead to activation of the NO-cGMP pathway in the CNS, contributing to the induction and maintenance of the hypoxia-induced increased ventilation (15). The importance of NO can be demonstrated by inhibition of the effects of NO (25) using L-arginine analogs such as L-NAME. In the present study we have chosen L-NAME because it is a nonselective inhibitor of NOS and acts on both the constitutive and inducible isoforms of the enzymes.

The present study confirms that hypoxiainduced hypothermia depends on the $\mathrm{NO}$ pathway since L-NAME injection abolished hypoxia-induced hypothermia in control and sham-operated rats. Also, NO may be involved in hypoxia-induced hyperventilation since control animals which received an injection of L-NAME had no ventilatory response to hypoxia (Figure 8). Moreover, LCLR which received an injection of LNAME showed a significantly lower hypoxia-induced hyperventilation compared to LCLR treated with saline, indicating that increased hypothermia-induced hyperventilation of LCLR may depend on the NO pathway in the CNS. In the present study, LC lesion caused no significant change in pulmonary ventilation or in body temperature under normoxic conditions, indicating that the LC plays no tonic role under control conditions. Conversely, hypoxia-induced hyperventilation was greatly increased in the LCLR, which may be related to the observed reduction in the hypoxia-induced hypothermia. Accordingly, previous studies have re- 
ported that the LC is important in the modulation of sensory processing by the brain and is activated by a variety of stressful somatic and autonomic stimuli $(26,27)$, but not during resting conditions (14). Activity in LC neurons is highest during wakefulness, particularly under conditions requiring increased alertness (28). The major component of adaptive responses to hypoxia is the stimulation of the sympathoadrenal system $(9,11)$, and sympathetic neurons are under the control of noradrenergic cell groups located in the brainstem (12), where the LC is the major noradrenergic nucleus. Recently, it has been reported that noradrenergic LC neurons send axons to spinal motoneurons, where they may participate in the control of respiratory movements (29). Changes in the activity of LC neurons would then be expected to elicit widespread effects in the CNS, including those of a respiratory nature. A study demonstrating the distribution of neuronal pathways activated by hypoxia reported that the LC presents an increased $c$-fos staining during hypoxia (30). Also, Pérez et al. (31) observed LC-mediated inhibition of chemosensory responses in the rat NTS, which is the zone of termination of afferents from baroreceptors and chemoreceptors travelling in the carotid sinus (32) and aortic sinus (33). Furthermore, Moore et al. (34) observed that LC cooling blocks the fall in respiratory output during hypoxia in anesthetized neonatal sheep. They concluded that the fall in the biphasic respiratory response is mediated by the activation of neurons inhibitory to respiratory output and involves either axons of passage or cell bodies lying in the LC region. In agreement with these data, the present study suggests that if there is a CNS inhibitory mechanism of hypoxiainduced hyperventilation, it seems to depend on neuronal function within the LC.

As to the ventilatory response, $\mathrm{LC}$ seems to be involved in modulation of tidal volume, since the hyperventilation response during hypoxia of LCLR differed from the control group due to a significant increase in tidal volume and not in respiratory frequency.

In summary, the present results suggest that $\mathrm{LC}$ and $\mathrm{NO}$ pathway may participate in the control of body temperature and pulmonary ventilation under hypoxic conditions, and that the inhibitory role of the LC on hypoxia-induced hyperventilation and its excitatory role in hypoxia-induced hypothermia may depend on the NO pathway.

\section{Acknowledgments}

We thank Mauro Ferreira Silva, Nadir Martins Fernandes and Rute Aparecida de Freitas Marcon for excellent technical assistance.

\section{References}

1. Lawson EE, Richter DW, Ballantyne D \& Lalley PM (1989). Peripheral chemoreceptor inputs to medullary inspiratory and postinspiratory neurons of cat. Pflügers Archive, 414: 523-533.

2. Völker A, Ballanyi K \& Richter DW (1995). Anoxic disturbance of the isolated respiratory network of neonatal rats. Experimental Brain Research, 103: 9-19.

3. Ramirez J M, Quellmalz UJ A, Wilken B \& Richter DW (1998). The hypoxic responses of neurons within the in vitro mammalian respiratory network. J ournal of Physiology, 507: 571-582.
4. Kawai $A$, Ballantyne $D$, Mükenhoff $K \&$ Scheid P (1996). Chemosensitive medullary neurons in the brainstem-spinal cord preparation of the neonatal rat. J ournal of Physiology, 492: 277-292.

5. Wood SC (1995). Oxygen as a modulator of body temperature. Brazilian J ournal of Medical and Biological Research, 28: 1249-1256.

6. Branco LGS \& Malvin GM (1996). Thermoregulatory effects of cyanide and azide in the toad, Bufo marinus. American J ournal of Physiology, 270: R169-R173.

7. Gautier H \& Murariu C (1998). Neuro- modulators and hypoxic hypothermia in the rat. Respiration Physiology, 112: 315324.

8. Hicks J W \& Wood SC (1985). Temperature regulation in lizards: effects of hypoxia. American J ournal of Physiology, 248: R595-R600.

9. Dalmaz $Y$, Pequignot J $M$, Cotted-Emard J M, Tavitian E \& Peyrin L (1989). Changes of dopamine, norepinephrine and epinephrine turnover in sympathetic tissues after long term hypoxia stress. In: Van Loon GR, Kvetnansky R, McCarty RM \& Axelrod J (Editors), Stress: Neurochemi- 
cal and Humoral Mechanisms. Gordon and Breach, New York, 777-785.

10. Marshall J M (1987). Analysis of cardiovascular responses evoked following changes in peripheral chemoreceptor activity in the rat. J ournal of Physiology, 394: 385-403.

11. Richalet J P (1990). The heart and adrenergic system in hypoxia. In: Sutton J R, Coatesand $G \& \&$ Remmers JE (Editors), Hypoxia: the Adaptations. Dekker, Toronto, 231-240.

12. Soulier V, Cottet-Emard J M, Pequignot J, Hanshin F, Peyrin L \& Pequignot J M (1992). Differential effects of long-term hypoxia on norepinephrine turnover in brain stem cell groups. J oumal of Applied Physiology, 73: 1810-1814.

13. Yang JJ, Chou YC, Lin MT \& Chiu TH (1997). Hypoxia-induced differential electrophysiological changes in rat locus coeruleus neurons. Life Sciences, 61: 17631773.

14. Breen S, Rees S \& Walker D (1996). Identification of brainstem neurons responding to hypoxia in fetal and newborn sheep. Brain Research, 748: 107-121.

15. Haxhiu MA, Chang $\mathrm{CH}$, Dreshaj IA, Erokwu BNR \& Prabhakar Cherniack NS (1995). Nitric oxide and ventilatory response to hypoxia. Respiration Physiology, 101: 257-266.

16. Scammell TE, Elmkist JK \& Saper CB (1996). Inhibition of nitric oxide synthase produces hypothermia and depresses lipopolysaccharide fever. American J ournal of Physiology, 271: R333-R338.

17. Branco LGS, Cámio EC \& Barros RCH (1997). Role of nitric oxide pathway in hypoxia-induced hypothermia of rats. American J ournal of Physiology, 273: R967-R971.

18. Paxinos G \& Watson C (1986). The Rat
Brain in Stereotaxic Coordinates. 2nd edn. Academic Press, San Diego.

19. Palkovits M \& J acobowitz DM (1974). Topographic atlas of catecholamine and acetylcholinesterase-containing neurons in the rat brain. II. Hindbrain (mesencephaIon, rhombencephalon). J ournal of Comparative Neurology, 157: 29-42.

20. Bartlett DJ \& Tenney SM (1970). Control of breathing in experimental anemia. Respiration Physiology, 10: 384-395.

21. Malan A (1973). Ventilation measured by body plethysmography in hibernating mammals and in poikilotherms. Respiration Physiology, 17: 32-44.

22. Teppema LJ , Veening J G, Kranenburg A, Dahan A, Berkenbosch A \& Olievier C (1997). Expression of c-fos in the rat brainstem after exposure to hypoxia and to normoxic and hyperoxic hypercapnia. J ournal of Comparative Neurology, 388: 169-190.

23. Wood SC (1991). Interaction between hypoxia and hypothermia. Annual Review of Physiology, 53: 71-85.

24. Moncada S, Palmer RMJ \& Higgs EA (1991). Nitric oxide: physiology, pathophysiology and pharmacology. Pharmacological Reviews, 43: 109-142.

25. Rees D, Palmer RMJ , Schultz R, Hodson H \& Moncada S (1990). Characterization of three inhibitors of endothelial nitric oxide synthase in vitro and in vivo. Brazilian J ournal of Pharmacology, 101: 746-752.

26. Aston-J ones G, Shipley MT, Chouvet G, Ennis M, Van Bockstaele E, Pieribone V, Shiekattar R, Akaoka H, Drolet G \& Astier $A B$ (1991). Afferent regulation of locus coeruleus neurons: Anatomy, physiology and pharmacology. Progress in Brain Research, 88: 47-75.

27. Van Bockstaele EJ, Akaoka H \& AstonJ ones G (1995). Integration in the ventral medulla and coordination of sympathetic, pain and arousal functions. Clinical and Experimental Hypertension, 17: 153-165.

28. Aston-J ones G, Foote SL \& Bloom FE (1984). Anatomy and physiology of locus coeruleus neurons: Functional implications. In: Ziegler M \& Lake CR (Editors), Norepinephrine (Frontiers of Clinical Neuroscience). Vol. 2. Williams \& Wilkins, Baltimore, 92-116.

29. Fung SJ , Chan J Y, Manzoni D, White SR, Lai YY, Strahlendorf HK, Zhuo H, Liu RH, Reddy VK \& Barnes CD (1994). Cotransmitter mediated locus coeruleus action on motoneurons. Brain Research Bulletin, 35: 423-432.

30. Nitsos I \& Walker DW (1999). The distribution of FOS-immunoreactive neurons in the brainstem, midbrain and diencephalon of fetal sheep in response to acute hypoxia in mid and late gestation. Brain Research, Developmental Brain Research, 114: 9-26.

31. Pérez $\mathrm{H}$, Ruiz $\mathrm{S}$, Laurido $\mathrm{C} \&$ Hernández $\mathrm{A}$ (1998). Locus coeruleus-mediated inhibition of chemosensory responses in the rat nucleus tractus solitarius is mediated by alpha2-adrenoreceptors. Neuroscience Letters, 249: 37-40.

32. Erickson J T \& Milhorn DE (1991). Fos-like protein is induced in neurons of the medulla oblongata after stimulation of the carotid sinus nerve in awake and anesthetized rats. Brain Research, 567: 11-24.

33. Ciriello J (1983). Brainstem projections of aortic baroreceptor afferent fibers in the rat. Neuroscience Letters, 36: 37-42.

34. Moore PJ, Ackland GL \& Hanson MA (1996). Unilateral cooling in the region of locus coeruleus blocks the fall in respiratory output during hypoxia in anaesthetized neonatal sheep. Experimental Physiology, 81: 983-994. 